\title{
Quick Identification of Stego Signatures in Images Using Suspicion Value (Special Reference to Concentrating Stego Algorithms)
}

\author{
Kaustubh Choudhary \\ Scientist, Defence Research and Development Organisation (DRDO), Naval College of Engineering, Indian \\ Naval Ship Shivaji, Lonavla, Maharashtra, India
}

\begin{abstract}
Steganography is a technique of hiding secret messages in the image in such a way that no one apart from the sender and intended recipient suspects the existence of the message. Image Steganography is frequently used by Terrorists and criminal organizations for securely broadcasting, dead-dropping and communicating the secret information over the internet by hiding it in the Images. As a result it becomes the most preferred tool for achieving secure CIA (Confidentiality, Integrity and Availability) compliant communication network capable of penetrating deep inside the civilian population. Steganalysis of Image (Identification of Images containing Hidden Information) is a challenging task due to lack of Efficient Algorithms, High rates of False Alarms and most importantly due to the High Computation Costs involved in Analyzing the Images. In this paper a computationally fast mathematical algorithm is designed for analyzing any stego-image generated using Spatial Domain Steganographic Algorithms and assigning it a Suspicion Value. This Suspicion Value is a number associated with the image and is bigger for those images which contain information and smaller for innocent images. Based on this Suspicion Value it can be decided whether the given image must be interrogated thoroughly (thorough interrogation of an image requires large amount of computation time but reliably and conclusively identifies the hidden information using various steganalytic techniques) or not. This fast stego-identifier algorithm can be used for quick filtering of the suspicious images flowing through the web servers, routers, layer three switches and any other electronic media. Based on this algorithm the suspicion values of various cover and stego-images generated by three different steganographic algorithms are computed and evaluated. All the graphs and tables are generated using MATLAB OImage Processing Tool Box.
\end{abstract}

Key Words: Bit Plane Slicing, Cyber Crime, Distributing Steganographic Algorithms. Global Terrorism, Image Steganalysis, Multicolor LSB Transform, Pixel Aberration, SDT based Image Steganography, Suspicion Value.

\section{INTRODUCTION}

Image based steganography is a dangerous technique of hiding secret messages in the image in such a way that no one apart from the sender and intended recipient suspects the existence of the message. It is based on invisible communication and this technique strives to hide the very presence of the message itself from the observer. As a result it has been used more frequently by various criminal and terrorist organizations than anybody else.[1][2][3] Various agencies even claim that 9/11 attacks have been masterminded and planned using image based steganography.[4] Image Steganography offers numerous advantages to the terrorists like Anonymity, Electronic Dead Dropping, Secure Broadcasting and above all very high Secrecy and Security (explained in detail in Section 5.2.1 of [5]). Thus an innocent looking digital image on any Web Portal, Online Auction Site or even a Social Networking Site may be probably hiding a malicious and deadly terrorist plan or any other significant criminal Information. The Steganalysis is the technique of identifying such malicious Stego-images (original image which is used for hiding data is called the Cover-Image whereas the image obtained after inserting the Secret Information in it is called Stego Image) from the bulk of innocent images. The next step of steganalysis involves either the extraction of the hidden information or destroying the information by adding visually imperceptible noise in the image or can be even used for embedding counter-information in the Stego-Image. Considering the voluminous bulk of images flowing every day through the Internet and amount of time and Computation Cost required for analyzing the Image the very first step of identifying an innocent looking Image as a Stego Image becomes the most challenging part of any Steganalysis procedure. It is because we do not have any foolproof method for crisply identifying a steganographic signature in the innocent looking stego-image.

Every steganographic algorithms has a different signature. As mentioned in [7][8] the most spatial domain steganographic algorithms can be broadly classified in to two types- Distributing Steganographic Algorithms and Concentrating Steganographic Algorithms. In other words all spatial domain steganographic 
Quick Identification of Stego Signatures in Images using Suspicion Value (special reference to

algorithms either embeds the information in the Least Significant Bits of the pixel or changes the entire color code of the pixel by inserting information in more than 2 bits of the pixel. In former algorithms large number of pixels are required for inserting information because only one or two LSB is available from every pixel and hence known as Distributing Type while in the latter algorithm the entire information can be stored in very few pixels because large numbers of bits are available from every pixel for storing information and hence called as Concentrating Type.

Since the Suspicion Value related with the Distributing Steganographic algorithms (Termed as Distributing Suspicion Value and represented by $\boldsymbol{\Lambda}$ ) is already calculated in [8]. So in this paper the suspicion value related with the Concentrating Steganographic algorithms is being determined. This suspicion value (related with concentrating stego algorithms) is here onwards termed as Concentrating Suspicion Value and represented by $\boldsymbol{\Gamma}$ in this entire paper. Based on this suspicion value (i.e. concentrating suspicion value $\boldsymbol{\Gamma}$ ) calculated in this paper and the distributing suspicion value $\boldsymbol{\Lambda}$ (determined in [8]) an overall suspicion value $\boldsymbol{\zeta}$ for any given image is calculated. This overall suspicion value $\zeta$ for any image is the holistic measure of the presence of information hidden using any Spatial Domain Stego Algorithm (i.e. Concentrating as well as Distributing algorithms) in the image and is termed as Spatial Domain Suspicion Value and represented here by as $\zeta$ in this entire paper.

\section{PRELIMINARIES FOR DETERMINATION OF CONCENTRATING SUSPICION VALUE ( $\Gamma)$}

The fast mathematical stego-identifier algorithm designed in this paper analyses any given digital image (for the presence of Concentrating spatial domain steganographic signatures) and quickly generates a Numerical Value (called in this text as Concentrating Suspicion Value and denoted by $\boldsymbol{\Gamma}$ ) corresponding to every image it has analyzed. This Suspicion Value is a number which is greater for those images which are more likely to have stego information and lower for innocent images.

\subsection{Preliminaries and Definition}

Before we proceed to the technique of generating the Concentrating Suspicion Value $\boldsymbol{\Gamma}$ for any image we have to mathematically define the preliminary concepts to be used in this model. These preliminary concepts are derived from the concepts mentioned in [6] and [7].

\section{Definition 1 (Image)}

Every digital image is collection of discrete picture elements or pixels. Let $\mathrm{M}$ be any digital image with $\mathrm{N}$ pixels. So any particular pixel of image $\mathrm{M}$ is represented as $\mathrm{M}(\mathrm{z})$ and $\mathrm{z}$ can be any value from 1 to $\mathrm{N}$. This $\mathrm{M}(\mathrm{z})$ can be a gray level intensity of the pixel in gray scale image or RGB or YCbCr value of the pixel in a color Image. The the individual RGB components of the pixel $M(z)$ in image $M$ is represented as $M^{R}(z), M^{G}(z)$ and $\mathrm{M}^{\mathrm{B}}(\mathrm{z})$ respectively. Thus $\mathrm{M}(\mathrm{z})$ can be a set $\left\{\mathrm{M}^{\mathrm{R}}(\mathrm{z}), \mathrm{M}^{\mathrm{G}}(\mathrm{z}), \mathrm{M}^{\mathrm{B}}(\mathrm{z})\right\}$ or equivalent gray scale representation or $\left(\mathrm{M}^{\mathrm{R}}(\mathrm{z})+\mathrm{M}^{\mathrm{G}}(\mathrm{z})+\mathrm{M}^{\mathrm{B}}(\mathrm{z})\right) / 3$. But it is always better to consider each $\mathrm{R}, \mathrm{G}$ and $\mathrm{B}$ components individually because the averaging effect cause loss of vital steganographic information. Further $\langle\{M\}, m>$ is multiset of

Image $\mathrm{M}$ such that $\mathrm{M}(\mathrm{z}) \in\{\mathrm{M}\}$ for every $\mathrm{z}=1$ to $\mathrm{N}$ and $\mathrm{m}$ is a vector corresponding to the occurrence or count of every element $M(z)$ in $\{M\}$. Mathematically an image $M$ with $N$ pixels is explained in (1)

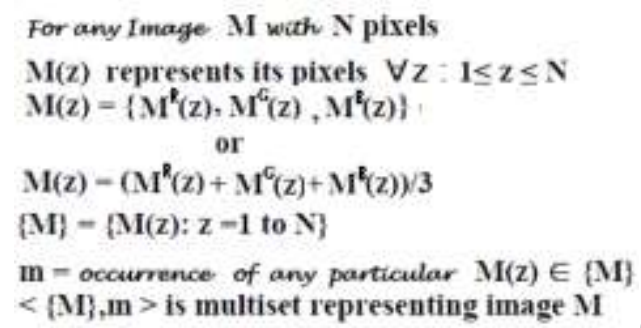

Definition 2 (Cardinality or Size of Image)

Any Image $M$ consists of certain number of pixels. So any particular pixel of image $M$ is represented as $\mathrm{M}(\mathrm{z})$ and $\mathrm{z}$ can be any value from 1 to total number of pixels in the image. The cardinality or the size of the image $M$ is the total number of pixels present in the image and represented as $n(M)$. So any Image $M$ has $n(M)$ pixels.

$$
\mathbf{M}=\bigcup_{\mathbf{z}=1}^{\mathbf{n}(\mathbf{M})} \mathbf{M}(\mathbf{z})
$$

Definition 3 (Component of an Image)

Any sub part of an Image is a component of the image. In other words any Image $\mathrm{M}$ can be broken down into pixel groups (or clusters) and each such cluster forms a component of the image and is identified by 
Quick Identification of Stego Signatures in Images using Suspicion Value (special reference to

its unique set of pixels. Thus in a Image M the Pixels M(z) from $z=1$ to $n(M)$ are the elements of the image M and the subsets of the image $M$ are composed of some of those pixels ( $M(z)$ from $z=1$ to $n(M)$ ) and thus forms the components of the image. Thus if the image $\mathrm{M}$ is broken in to $\mathrm{K}$ components then any component $\mathrm{M}^{\mathrm{i}}$ of the image $\mathrm{M}$ is mathematically explained as:

$$
\begin{aligned}
& \mathrm{M}^{\mathrm{i}} \subset \mathrm{M} \text { and } \forall \mathbf{i}: \mathbf{1} \leq \mathbf{i} \leq \mathrm{K} \\
& M=\bigcup_{\substack{M^{i} \subset M \\
\forall i: 1 \leq i \leq K}} M^{i}
\end{aligned}
$$

Also for every component $M^{i}$ of the image $M$ the $M^{i}(z)$ represents the pixels of the component $M^{i}$ and $n\left(M^{i}\right)$ represents the number of pixels in $\mathrm{M}^{\mathrm{i}}$.

\section{Definition 4 (Neighborhood or Locality of Pixel)}

If $\ell(M(z))$ is said to be set of neighboring pixels of any pixel $M(z)$ in image $M$. Then any $n_{i} \in \ell(M(z))$ will be such that $d\left(\mathrm{n}_{\mathrm{i}}, \mathrm{M}(\mathrm{z})\right) \leq \lambda$ where $d$ is a function which calculates distance (can be Euclidean, City-Block, Chess Board or any other type depending upon the steganographic algorithm) between its inputs (ie $n_{i}$ and $\mathrm{M}(\mathrm{z})$ ) and $\lambda$ is measurement of degree of neighbourhood and should be minimum (Generally equal to 1 pixel) but also depends upon the steganographic algorithm used. Mathematically this can be represented as:

$$
\left.\mathrm{I}(\mathrm{M}(\mathrm{z}))=\{\mathrm{M}(\mathrm{x}): \mathrm{M}(\mathrm{x}) \in \mathrm{M} \text { and } d(\mathrm{M}(\mathrm{x}), \mathrm{M}(\mathrm{z})) \leq \lambda\}_{4}\right)
$$

In Fig 1 an arbitrary pixel $\mathrm{Y}$ is shown with its immediate neighbors $\mathrm{P}, \mathrm{Q}, \mathrm{R}, \mathrm{S}, \mathrm{T}, \mathrm{U}, \mathrm{V}$ and $\mathrm{W}$. We represent this pixel $\mathrm{Y}$ as $\dot{\mathrm{Y}}$ in mathematical notation. Thus $\ell(\dot{\mathrm{Y}})=\{\mathrm{P}, \mathrm{Q}, \mathrm{R}, \mathrm{S}, \mathrm{T}, \mathrm{U}, \mathrm{V}, \mathrm{W}\}$ is set of neighboring pixels of pixel $\mathrm{Y}$. Here $\lambda=1$ and distance function $d$ calculates Chess Board Distance.

\begin{tabular}{|l|l|l|}
\hline $\mathrm{P}$ & $\mathrm{Q}$ & $\mathrm{R}$ \\
\hline $\mathrm{S}$ & $\mathrm{Y}$ & $\mathrm{T}$ \\
\hline $\mathrm{U}$ & $\mathrm{V}$ & $\mathrm{W}$ \\
\hline
\end{tabular}

Fig 1 Pixel $Y$

Definition 5 (Adjacent Neighbors of Pixel)

Set of Adjacent Neighbors of a pixel M(z) is given as $\mathcal{A}(\mathrm{M}(\mathrm{z}))$. Thus $\mathcal{A}(\mathrm{M}(\mathrm{z}))$ is a collection of set $\{\mathrm{M}(\mathrm{x}), \mathrm{M}(\mathrm{y})\}$ such that $\mathrm{M}(\mathrm{x}) \in \ell(\mathrm{M}(\mathrm{z}))$ and $\mathrm{M}(\mathrm{y}) \in \ell(\mathrm{M}(\mathrm{z}))$ and they are adjacent i.e $d(\mathrm{M}(\mathrm{x}), \mathrm{M}(\mathrm{y}))=1$ where $d$ is a function which calculates distance. Mathematically:

$$
\begin{aligned}
& \mathcal{A}(\mathrm{M}(\mathrm{z}))=\{\{\mathrm{M}(\mathrm{x}), \mathrm{M}(\mathrm{y})\}: \mathrm{M}(\mathrm{x}) \in \mathrm{f}(\mathrm{M}(\mathrm{z})) \\
& \text { and } \mathrm{M}(\mathrm{y}) \in \mathcal{l}(\mathrm{M}(\mathrm{z})) \text { and } d(\mathrm{M}(\mathrm{x}), \mathrm{M}(\mathrm{y}))=1\}_{(5)}
\end{aligned}
$$

In Fig 1 for an arbitrary pixel $\mathrm{Y}$ with $\ell(\dot{\mathrm{Y}})=\{\mathrm{P}, \mathrm{Q}, \mathrm{R}, \mathrm{S}, \mathrm{T}, \mathrm{U}, \mathrm{V}, \mathrm{W}\}$ the $\mathcal{A}(\dot{\mathrm{Y}})=\{\{\mathrm{P}, \mathrm{Q}\},\{\mathrm{Q}, \mathrm{R})\}$, $\{\mathrm{R}, \mathrm{T}\},\{\mathrm{T}, \mathrm{W}\},\{\mathrm{W}, \mathrm{V}\},\{\mathrm{V}, \mathrm{U}\},\{\mathrm{U}, \mathrm{S}\},\{\mathrm{S}, \mathrm{P}\}\}$.

\section{Definition 6 (Pixel Aberration)}

Pixel Aberration of any Pixel M(z) is the measure of the degree of difference of the given pixel M(z) from its immediate neighborhood i.e. $\ell(\mathrm{M}(\mathrm{z})$ ): $\mathrm{d}$ : $\Leftrightarrow$ Chess Board Distance $\wedge \lambda=1$ (immediate neighborhood is obtained when Neighborhood or Locality Function $\ell(\mathrm{M}(\mathrm{z}))$ is calculated with $\lambda=1$ and distance function $\mathrm{d}$ is used for determining Chess Board Distance) and represented as $\delta(\mathrm{M}(\mathrm{z}), \ell(\mathrm{M}(\mathrm{z}))$. It is measured in terms of Standard Deviation of $\ell(\mathrm{M}(\mathrm{z}))$ and acts like a quantifier which gives the idea of the amount of deviation of the pixel from its immediate neighborhood.

Basic concept used for determining the pixel aberration of any pixel is based on the fact that, in any natural image a pixel $\mathrm{M}(\mathrm{z})$ is expected to be as much different from its immediate neighborhood i.e. $\ell(\mathrm{M}(\mathrm{z}))$ as the adjacent pairs of pixels in $\ell(\mathrm{M}(\mathrm{z}))$ themselves are. The same concept is explained in (6). Using simple statistical techniques the concept developed in (6) is applied for determining the value of Pixel Aberration for any Pixel in any given image. For any pixel $\mathrm{M}(\mathrm{z})$ in image $\mathrm{M}$ the mean of its absolute difference from its immediate neighborhood $\ell(\mathrm{M}(\mathrm{z}))$ is given as $\overline{(M(z), \ell(M(z)))}$. And the set representing the absolute differences of the adjacent neighbors of $\mathrm{M}(\mathrm{z})$ among themselves is given as $\mathcal{D}(\mathcal{A}(\mathrm{M}(\mathrm{z})))$. The mean of the values of $\mathcal{D}(\mathcal{A}(\mathrm{M}(\mathrm{z})))$ is given as $\overline{D(\mathcal{A}(M(z)))}$ and Standard Deviation of the values of $\mathcal{D}(\mathcal{A}(\mathrm{M}(\mathrm{z})))$ is given as $\sigma(\mathcal{D}(\mathcal{A}(\mathrm{M}(\mathrm{z}))))$. Since $\mathrm{M}(\mathrm{z})$ is also a immediate neighbor of every pixel in $\ell(\mathrm{M}(\mathrm{z}))$ so $\overline{(M(z), \ell(M(z)))}$ must be within the limits of standard deviation of $\mathcal{D}(\mathcal{A}(\mathrm{M}(\mathrm{z})))$ (represented as $\sigma(\mathcal{D}(\mathcal{A}(\mathrm{M}(\mathrm{z}))))$ ) and mean of $\mathcal{D}(\mathcal{A}(\mathrm{M}(\mathrm{z})))$ (represented as $\overline{D(\mathcal{A}(M(z)))})$. This degree of deviation of $\mathrm{M}(\mathrm{z})$ from its neighbors $\ell(\mathrm{M}(\mathrm{z}))$ in 
Quick Identification of Stego Signatures in Images using Suspicion Value (special reference to terms of $\sigma(\mathcal{D}(\mathcal{A}(\mathrm{M}(\mathrm{z}))))$ and $\overline{D(\mathcal{A}(M(z)))}$ is quantified as pixel aberration of pixel $\mathrm{M}(\mathrm{z})$ and represented as $\delta(\mathrm{M}(\mathrm{z}), \ell(\mathrm{M}(\mathrm{z})))$.

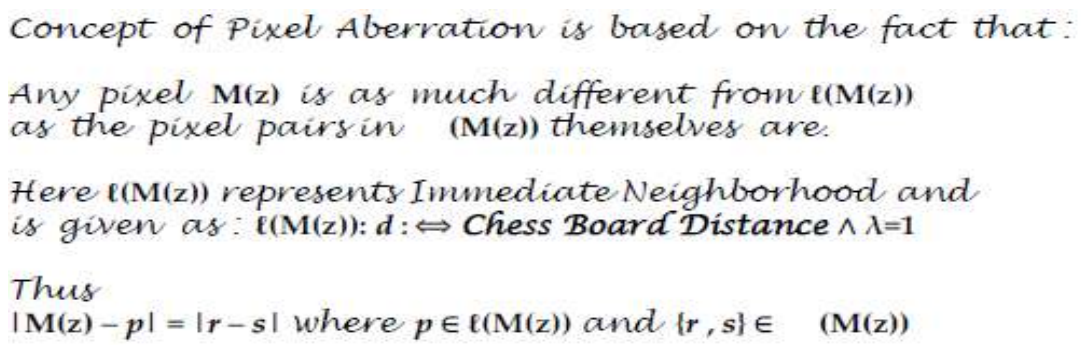

In terms of Fig 1 the mean of the differences of pixel $Y$ with its neighbors i.e. elements of $\ell(\dot{Y})$ is given as Y-P,Y-Q, Y-R, Y-S, Y-T, Y-U, Y-V and Y-W and should be close to the differences of the adjacent pixels in $\ell(\dot{Y})$ or in other words the difference of the pixel pairs in $\mathcal{A}(\dot{Y})$ i.e. difference of the elements of the pixel pairs $\{\mathrm{P}, \mathrm{Q}\},\{\mathrm{Q}, \mathrm{R})\},\{\mathrm{R}, \mathrm{T}\},\{\mathrm{T}, \mathrm{W}\},\{\mathrm{W}, \mathrm{V}\},\{\mathrm{V}, \mathrm{U}\},\{\mathrm{U}, \mathrm{S}\}$ and $\{\mathrm{S}, \mathrm{P}\}$ or simply $\mathrm{P}-\mathrm{Q}, \mathrm{Q}-\mathrm{R}, \mathrm{R}-\mathrm{T}, \mathrm{T}-\mathrm{W}, \mathrm{W}-\mathrm{V}, \mathrm{V}-\mathrm{U}$, U-S and S-P. Thus $((\dot{Y}), \ell((\dot{Y})))$ is mean of modulus of Y-P, Y-Q, Y-R, Y-S, Y-T, Y-U, Y-V and Y-W and $\mathcal{D}(\mathcal{A}(\dot{\mathrm{Y}}))=\{$ modulus of P-Q, Q-R, R-T, T-W, W-V, V-U, U-S and S-P $\}$. So aberration in pixel Y with respect to its neighborhood $\ell(\dot{\mathrm{Y}})$ given as $\delta(\dot{\mathrm{Y}}, \ell(\dot{\mathrm{Y}}))$ should be within the limits of standard deviation of $\mathcal{D}(\mathcal{A}(\dot{\mathrm{Y}}))$ and it mean $\overline{D(\mathcal{A}(Y))}$.

Mathematically:

$$
\begin{aligned}
& \overline{(M(z), \mathcal{\ell}(M(z)))}=\frac{1}{n(\ell(M(z)))} \sum_{p \in\{(M(z))}|p-M(z)| \\
& \mathcal{D}(\mathcal{A}(\mathrm{M}(\mathrm{z})))=\{|\mathbf{x}-\mathbf{y}|:(\mathbf{x}, \mathbf{y}) \in \mathcal{A}(\mathbf{M}(z))\} \\
& \delta(\mathrm{M}(\mathrm{z}), \ell(\mathrm{M}(\mathrm{z})))=\frac{\overline{(M(z),[(M(x)))}-\overline{\sigma(A(M(z)))}}{\sigma(\mathrm{D}(\mathcal{A}(\mathrm{M}(\mathrm{z}))))} \text { (7) }
\end{aligned}
$$

Definition 7 (Pixel Aberration of the Entire Image (Weighted Mean))

In any image $\mathrm{M}$ with $\mathrm{N}$ pixels (i.e. $\mathrm{n}(\mathrm{M})=\mathrm{N}$ ) the Pixel aberration of image $\mathrm{M}$ is given as $\delta(M)$. It is a quantifier whose high values for any given image $M$ indicates that relatively large number of pixels in $M$ have high pixel aberration. It is calculated by determining the weighted mean of the modulus of the pixel aberrations of the pixels of the entire image $\mathrm{M}$.

Since for any image $\mathrm{M}$ the $\delta(\mathrm{M}(\mathrm{z}), \ell(\mathrm{M}(\mathrm{z})))$ is the measure of deviation of $\mathrm{M}(\mathrm{z})$ from its neighborhood $\ell(\mathrm{M}(\mathrm{z}))$ in terms of standard deviation so majority of pixels have this values located close to zero and approximately more than $68 \%$ of the pixels have pixel aberration within \pm 1 ( as per 3 Sigma or 68-95-99.7 rule of Statistics). Hence the simple mean of $\delta(\mathrm{M}(\mathrm{z}), \ell(\mathrm{m}(\mathrm{z})))$ is very close to zero and is insignificantly small for all images. Since by pixel aberration analysis we have to identify those images which have larger pixel aberrations so as a remedy very small weights are assigned to less deviated values (majority of pixels which have low pixel aberration values) and larger weights are assigned to more deviated values (few counted pixels have large pixel aberrations). Thus value of $\delta(M)$ for the Image $\mathrm{M}$ with $\mathrm{N}$ pixels is given as:

$$
\delta(M)=\frac{\sum_{\substack{t=1 \\ M(z) \in \mathcal{N}}}^{N}|\delta(\mathrm{M}(\mathrm{z}), \mathrm{t}(\mathrm{M}(\mathrm{z})))| \times W(z)}{\sum_{\substack{z=1 \\ M(z) \in M}}^{N} W(z)}
$$

Where the weight $\mathrm{W}(\mathrm{z})$ for the pixel $\mathrm{M}(\mathrm{z})$ is very small for majority of the pixels (which have $\delta(\mathrm{M}(\mathrm{z}), \ell(\mathrm{m}(\mathrm{z})))$ value close to the mean value of the pixel aberration of all the pixels together in the image) and quite large for the pixels having highly deviated values of $\delta(\mathrm{M}(\mathrm{z}), \ell(\mathrm{m}(\mathrm{z})))$ (The value of $\delta(\mathrm{M}(\mathrm{z}), \ell(\mathrm{m}(\mathrm{z})))$ for such pixels is very different from the mean of $\delta(\mathrm{M}(\mathrm{z}), \ell(\mathrm{m}(\mathrm{z})))$ ) for all pixels together). Such weights (which are larger for pixels having greater pixel aberration (in absolute terms) and much smaller for pixels having lesser pixel aberration) can be computed by taking cube of the value of pixel 
Quick Identification of Stego Signatures in Images using Suspicion Value (special reference to aberration in terms of the standard deviation. In other words the weight $\mathrm{W}(\mathrm{z})$ for any Pixel $\mathrm{M}(\mathrm{z})$ in image $\mathrm{M}$ is given as

$$
\begin{aligned}
& W(\mathrm{z})=\left|\frac{\delta(\mathrm{M}(\mathrm{z}), \mathrm{\ell}(\mathrm{M}(\mathrm{z})))-\mathrm{MEAN}_{\mathrm{Z}=1}^{\mathrm{Z}=\mathrm{N}}(\delta(\mathrm{M}(\mathrm{z}), \mathrm{\ell}(\mathrm{M}(\mathrm{z}))))}{\operatorname{STD} \mathrm{Z}=\mathrm{N}(\delta(\mathrm{M}(\mathrm{z}), \mathrm{C}(\mathrm{M}(\mathrm{z}))))}\right|^{3}
\end{aligned}
$$

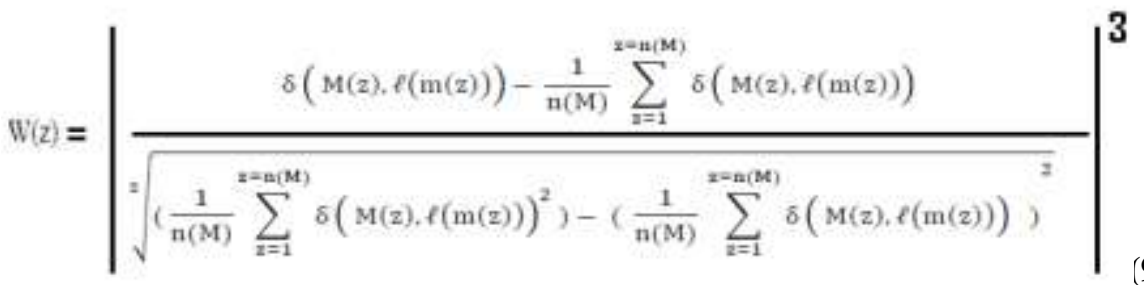

\subsection{Properties of Stego Image}

Properties of stego-images (images containing information) depends upon the properties of those pixels in the stego-image which are storing the information. In other words the properties of stego-images become different from the innocent image (image without information) due to deformation produced in certain pixels of stego-image due to embedding of information in those pixels. The basic concepts of steganalysis of Distributing Stego Algorithms is given in Section 3.2 of [7] and the concepts related to steganalysis of Concentrating StegoAlgorithms is given in Section 2.1 of [7] and Section 2.3.1 of [6] (Requirement 3 and Requirement 4).

From [6] and [7] it can be conclusively said that Information pixels (pixels containing hidden information) have following 4 main properties:

1. Since the information pixels are suffering deformations so they are generally quite different from their immediate neighbors. As a result the pixel aberration of information pixels is quite high. Since the concentrating algorithms bring bigger changes in the pixel so Pixel Aberration based analysis is more responsive to the steganalysis of Concentrating Stego-Algorithms.

2. Information has maximum concentration in the LSB Plane of the image. But the LSB Plane of any image appears black and hence its contrast is increased by obtaining the Multicolored LSB Transform of the image. Thus in the Multicolored LSB Transform we can clearly see the information pixels differently colored from the innocent pixels. But since concentrating algorithms change only few pixels and as pixels are very small in size so few counted modified pixels in the Multicolored LSB Plane are imperceptible to human eye and are also statistical point of view are insignificantly less in number. But this method applies perfectly well in steganalysis of Distributing Stego-Algorithms because they modify large number of pixels.

3. In any statistically significant component (50 x 50 pixels) of the Multicolored LSB Plane the distribution of Red, Green and Blue components is significantly unequal among information pixels where as they are nearly equal for innocent pixels. Thus the degree of deviation is more in the information pixels then the innocent pixels.

4. The information pixels are always present in the Fine Grained Pixel Clusters and rarely in the Coarse grained pixel clusters. They are always absent in the Continuous and Boulder Grained pixels Clusters. Refer Section 3.2 of [7] for details of the classification of pixel clusters. The Multi Color LSB Transform of the images with fine grained pixel clusters have majority of pixel with large value of Pixel Anomaly. Thus the value of the Mean Pixel Anomaly is largest in the fine grained pixel clusters and is lesser in coarse grained and even lesser in boulder grained and least in continuous grained pixel clusters.

\subsection{Quantification of the Properties of Stego Image generated by Concentrating Stego Algorithm using Pixel Aberration of the entire Image}

These 4 properties associated with the stego-image, can be quantified in to an equivalent numerical values corresponding to the given stego-image. The last three properties are associated with Distributing Stego Algorithms and hence were used in determining Distributing Suspicion Value $\boldsymbol{\Lambda}$ for any given image in [8]. By using the definitions given in Section 2.1 the first property (related with Pixel Aberration and associated with Concentrating Stego Algorithms) is used for determining the Concentrating Suspicion Value $\boldsymbol{\Gamma}$ for any given image. Both these numerical values $(\boldsymbol{\Lambda}$ and $\boldsymbol{\Gamma})$ when combined together will be used for determining the holistic Spatial Domain Suspicion Value $\boldsymbol{\zeta}$ associated with the image.

\subsubsection{Quantification of the Properties Using Weighted Mean Pixel Aberration}

Pixel Aberration based analysis responds well to all stego algorithms in general and Concentrating Stego Algorithms in Particular. The concept of Pixel Aberration is based on [6] and [7] and is explained in 
Quick Identification of Stego Signatures in Images using Suspicion Value (special reference to

detail in Definition 6 of Section 2.1 of this paper and mathematically represented in (6) and (7). Since the Pixel Aberration is based on standard deviation so majority of pixels have pixel aberration close to 0 . Thus in Definition 7 of Section 2.1 of this paper the pixel aberration for the entire image is calculated by determining the weighted mean of pixel aberrations of all the pixels in the given image and is mathematically represented in (8) and (9). Thus we examine the performance of Weighted Mean Pixel Aberration as given in (8) and (9) as the measure of Concentrating Suspicion Value $\boldsymbol{\Gamma}$ for any given image. For this purpose we use two different $100 \mathrm{x}$ 100 Pixel Images as the cover images. They are represented as A and B and shown in Fig 2. Three different stego algorithms are used for embedding same information (this entire paragraph consisting of 1610 Characters) in all the four images. The first two algorithms are of distributing type (named as Distributing Algorithm 1 and Distributing Algorithm 2) and the third is of Concentrating type. Also the Distributing Algorithm 1 embeds the secret information vertically (Column by Column) and Distributing Algorithm 2 embeds the secret message horizontally (row by row). The three steganographic algorithms used in this paper namely Distributing Algorithm 1, Distributing Algorithm 2 and Concentrating Algorithm were analyzed in [5] and are referred in Section 5 of [5] as Algorithm designed in section 4, QuickStego Software and Eureka Steganographer respectively. The stego-images obtained after inserting information from these three different algorithms are represented as A1, A2, A3 and B1, B2, B3 respectively. Here only A3 and B3 are stego images corresponding to Concentrating Algorithms and rest (A1, A2 and B1, B2) are obtained from Distributing Algorithms.

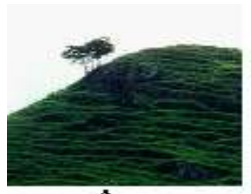

A

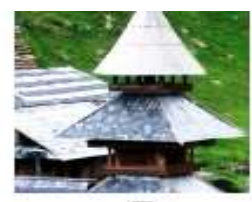

B

Fig $2100 \times 100$ Test Images

The Image B has the properties similar to most other images and hence is a perfect example of a general image but Image A represents a special case of rare occurring images. The Image A is selected because it is one such rare image which has pixel aberration in initial (Row by Row order) few pixels as almost zero. As a result all other pixels (which do not have Pixel Aberration as absolutely zero) get very high weights causing exceptionally high values of weighted mean pixel aberration for the entire image even though the mean (simple mean) pixel aberration for the pixels of entire image is relatively very low. The same is shown in Table 1 and Table 2. The values of Pixel Aberration for all these eight images (i.e. the Cover Images A and B and the corresponding stego images A1, A2, A3 and B1, B2, B3 ) are graphically shown in Fig 3. By using (6),(7),(8) and (9) the weighted mean pixel aberration and by using (11) the mean pixel aberration is calculated for all these eight images and the same is tabulated in Table 1 and Table 2 respectively.

Weighted Mean Pixel Aberration for any image M is represented as $\boldsymbol{\delta}_{\mathbf{w}}(\mathbf{M})$ but its value is different for all the three color components red, green and blue and hence the three color components are represented as $\boldsymbol{\delta}_{\mathbf{w}}{ }^{\mathbf{R}}(\mathbf{M}), \boldsymbol{\delta}_{\mathbf{w}}{ }^{\mathbf{G}}(\mathbf{M})$ and $\boldsymbol{\delta}_{\mathbf{w}}{ }^{\mathbf{B}}(\mathbf{M})$ respectively. The mean pixel aberration (mean of all the three color components) and Maximum of the three color components represented as $\boldsymbol{\delta}_{\mathbf{w}}{ }^{\text {MEAN }}(\mathbf{M})$ and $\boldsymbol{\delta}_{\mathbf{w}}{ }^{\text {MAX }}(\mathbf{M})$ respectively is also shown in Table 1. Also $\boldsymbol{\delta}_{\mathbf{w}}{ }^{\text {MAX }}(\mathbf{M})$ and $\boldsymbol{\delta}_{\mathbf{w}}{ }^{\text {MEAN }}(\mathbf{M})$ is explained mathematically in (10).

$$
\begin{aligned}
& \delta_{w}{ }^{M A X}(M)=\operatorname{MAX}\left(\delta_{w}^{R}(M), \delta_{w}^{G}(M), \delta_{w}{ }^{\mathrm{B}}(M)\right) \\
& \delta_{w}^{\text {MEAN }}(\mathbf{M})=\left(\delta_{w}^{\mathrm{R}}(\mathbf{M})+\delta_{w}^{\mathrm{G}}(\mathbf{M})+\delta_{\mathrm{w}}^{\mathrm{B}}(\mathbf{M})\right) / 3 \text {. }
\end{aligned}
$$

Also simple mean for any image M is represented by $\boldsymbol{\delta}_{\mathrm{m}}(\mathbf{M})$. Also $\boldsymbol{\delta}_{\mathrm{m}}(\mathbf{M})$ has 3 color components represented as $\boldsymbol{\delta}_{\mathrm{m}}{ }^{\mathbf{R}}(\mathbf{M}), \boldsymbol{\delta}_{\mathrm{m}}{ }^{\mathbf{G}}(\mathbf{M})$ and $\boldsymbol{\delta}_{\mathrm{m}}{ }^{\mathbf{B}}(\mathbf{M})$ and also the mean pixel aberration (mean of all the three color components) and Maximum of the three color components represented as $\boldsymbol{\delta}_{\mathbf{m}}{ }^{\text {MEAN }}(\mathbf{M})$ and $\boldsymbol{\delta}_{\mathbf{m}}{ }^{\text {MAX }}(\mathbf{M})$ respectively. The values of $\boldsymbol{\delta}_{\mathrm{m}}{ }^{\mathrm{R}}(\mathbf{M}), \boldsymbol{\delta}_{\mathrm{m}}{ }^{\mathrm{G}}(\mathbf{M})$ and $\boldsymbol{\delta}_{\mathrm{m}}{ }^{\mathbf{B}}(\mathbf{M}), \boldsymbol{\delta}_{\mathrm{m}}{ }^{\mathrm{MAX}}(\mathbf{M})$ and $\boldsymbol{\delta}_{\mathrm{m}}{ }^{\text {MEAN }}(\mathbf{M})$ for all these eight images is calculated using (11) and tabulated in Table 2.

$$
\delta_{m}(M)=\frac{1}{n(M)} \sum_{z=1}^{z=n(M)} \delta(M(z), \ell(M(z)))
$$

From Table 1 and Table 2 we can clearly see that even though the mean pixel aberration of image A is lower than the Image B (Table 2) but still the weighted mean pixel aberration for rare occurring Image A is many times higher (Table 1) than the regular image B (due to distortions in A as explained earlier). Thus we clearly see that determination of the overall pixel aberration by using weighted mean pixel aberration introduces certain unnecessary distortions in few images. Moreover the calculation of weighted pixel aberration requires determination of standard deviation of the pixel aberration for all the pixels of the images and becomes highly 
Quick Identification of Stego Signatures in Images using Suspicion Value (special reference to

complex and time consuming. In fact determination of weighted mean pixel aberration for large images (more than $200 \times 200$ Pixels) consumes very high computational costs and hangs the program on most occasions. But at the same time relying solely on the simple mean will also not yield a suitable result because for most large images its value becomes insignificantly small. As a remedy a technique based on combination of simple means and weighted means of the pixel aberration for entire image is used for determining the Concentrating Suspicion Value $\boldsymbol{\Gamma}$ for any given image.

Table 1

The values of Weighted Means of the Cover Inages A and B and the corresponding Stego Images

\begin{tabular}{|c|c|c|c|c|c|c|c|}
\hline $\begin{array}{l}\text { Image } \\
\text { M }\end{array}$ & Description of M & $\delta_{\mathrm{m}}^{\mathrm{R}}(\mathrm{M})$ & $\delta_{w}^{G}(M)$ & $\delta_{w}^{B}(M)$ & $\delta_{\mathrm{w}}^{\mathrm{M}-\mathrm{IX}}(\mathrm{M})$ & $\delta_{\mathrm{w}}^{\text {ME. }}{ }^{\mathrm{Y}}(\mathrm{MI})$ & Remarks \\
\hline$A$ & $\begin{array}{l}\text { Corer Image } \\
\text { (Fig 2, Left) }\end{array}$ & 18.7204 & 6.5554 & 7.9115 & 18.7204 & 11.0624 & \multirow{4}{*}{$\begin{array}{l}\text { Corer Image has } \\
\text { High } \delta_{\text {nr }}(\mathbf{M}) \text {. } \\
\text { Such images with } \\
\text { high ralue of } \\
\text { weighted mean } \\
\text { pixel aberration } \\
\text { occur rarely. }\end{array}$} \\
\hline Al & $\begin{array}{l}\text { Stege } \\
\text { (Distributing 1) }\end{array}$ & 45.5845 & 7.6738 & 218.4825 & 218.4825 & 90.5802 & \\
\hline$A 2$ & $\begin{array}{l}\text { Stego } \\
\text { (Distributing 2) }\end{array}$ & 18.1708 & 5.7362 & 7.5359 & 18.1708 & 10.4809 & \\
\hline$A 3$ & $\begin{array}{l}\text { Stege } \\
\text { (Concentrafing) }\end{array}$ & 18.5978 & 16.4445 & 16.5742 & 18.5978 & 17.2055 & \\
\hline B & $\begin{array}{l}\text { Cover Image } \\
\text { (Fig 2, Right) }\end{array}$ & 3.7040 & 4.1700 & 3.6797 & 4.1700 & 3.8512 & \multirow{4}{*}{$\begin{array}{l}\text { Cover Image has } \\
\text { Low } \delta_{w}(\mathrm{M}) \text {. } \\
\text { It represents } \\
\text { commonly } \\
\text { occurring general } \\
\text { every day image }\end{array}$} \\
\hline $\mathrm{Bl}$ & $\begin{array}{l}\text { Stego } \\
\text { (Distributing 1) }\end{array}$ & 3.3931 & 3.4228 & 3.7255 & 3.7255 & 3.5138 & \\
\hline B2 & $\begin{array}{l}\text { Stege } \\
\text { (Distributing 2) }\end{array}$ & 3.6591 & 4.3328 & 3.7444 & 4.3328 & 39221 & \\
\hline B3 & $\begin{array}{l}\text { Stego } \\
\text { (Concentrating) }\end{array}$ & 11.5535 & 14.1091 & 31.2673 & 31.2673 & 18.9766 & \\
\hline
\end{tabular}

Table 2

The values of Simple Means of the Cover Images A and B and the corresponding Stego Images

\begin{tabular}{|c|c|c|c|c|c|c|}
\hline Image $\mathbf{M}$ & Description of $\mathbf{M}$ & $\delta_{\text {m }}{ }^{R}(M)$ & $\delta_{\mathrm{m}}^{G}(\mathrm{M})$ & $\delta_{\mathrm{m}}^{\mathrm{B}}(\mathrm{M})$ & $\delta_{\text {m }}{ }^{M A X}(M)$ & $\delta_{\text {m }}^{\text {IIIAI }}(\mathrm{MI})$ \\
\hline A & $\begin{array}{l}\text { Cover Image } \\
\text { (Fig 2, Left) }\end{array}$ & 0.1136 & 0.1235 & 0.1045 & 0.1235 & 0.1139 \\
\hline Al & Stego (Distributing I) & 0.1298 & 0.1146 & 0.1035 & 0.1298 & 0.1159 \\
\hline A2 & Stego (Distributing 2) & 0.1121 & 0.1294 & 0.1071 & 0.1294 & 0.1162 \\
\hline$A 3$ & Stego (Concentrating) & 0.1597 & 0.1601 & 0.1488 & 0.1601 & 0.1562 \\
\hline B & $\begin{array}{l}\text { Cover Image } \\
\text { (Fig 2, Right) }\end{array}$ & 0.1842 & 0.1781 & 0.1742 & 0.1842 & 0.1788 \\
\hline B1 & Stego (Distributing I) & 0.1823 & 0.1762 & 0.1743 & 0.1823 & 0.1776 \\
\hline B2 & Stego (Distributing 2) & 0.1837 & 0.1767 & 0.1745 & 0.1837 & 0.1783 \\
\hline B3 & Stego (Concentrating) & 0.2049 & 0.2053 & 0.1997 & 0.2053 & 0.2033 \\
\hline
\end{tabular}

\subsubsection{Quantification of the Properties Using Combination of Simple and Weighted Mean Pixel}

\section{Aberration for Determination of Concentrating Suspicion Value}

There are two different possible approaches of combining simple mean and weighted mean together for calculation of the Concentrating Suspicion Value $\boldsymbol{\Gamma}$. Both these approaches are computationally fast because they use a variant of Divide and Conquer Technique and hence break the entire image into small $5 \times 5$ to $10 \times 10$ Pixel Components.

In the first approach the individual simple mean Pixel Aberration for each component is calculated. Using the values of the individual means the overall weighted mean for all the image components together can be calculated as the value of overall Pixel Aberration for any given Image and represented by $\boldsymbol{\delta}_{\mathbf{1}}(\mathbf{M})$ for any Image $\mathbf{M}$. The process of calculating $\boldsymbol{\delta}_{\mathbf{1}}(\mathbf{M})$ for any Image $\mathbf{M}$ is explained mathematically in (12). The concentrating suspicion value obtained using Pixel Aberration of any given Image obtained by first approach i.e. $\delta_{1}(\mathbf{M})$ is represented by $\boldsymbol{\Gamma}_{\mathbf{1}}(\mathbf{M})$ for any given image $\mathbf{M}$.

In second approach the weighted mean pixel aberration is calculated for all the pixels in each component and then an overall simple mean is calculated for weighted mean pixel aberration of each component. Pixel Aberration for any given Image obtained by this approach is represented as $\boldsymbol{\delta}_{\mathbf{2}}(\mathbf{M})$. The process of calculating $\boldsymbol{\delta}_{\mathbf{2}}(\mathbf{M})$ for any Image $\mathrm{M}$ is explained mathematically in (13). The concentrating suspicion value obtained using Pixel Aberration of any given Image obtained by second approach i.e. $\boldsymbol{\delta}_{2}(\mathbf{M})$ is represented by $\boldsymbol{\Gamma}_{2}(\mathbf{M})$ for any given image $\mathbf{M}$.

\subsubsection{Concentrating Suspicion Value Calculation by First Approach}

The algorithm for calculating the Pixel Aberration for any given Image by combining the Simple and Weighted Means together by First Approach is given in (12). On the basis of (12) the value of $\boldsymbol{\delta}_{\mathbf{1}}(\mathbf{M})$ is calculated for the same images (Fig 2) and shown in Table 3. Since the calculation of $\boldsymbol{\delta}_{\mathbf{1}}(\mathbf{M})$ uses Divide and Conquer technique so its computation is much faster than the calculation of plain weighted mean pixel aberration for entire image i.e. $\boldsymbol{\delta}_{\mathbf{w}}(\mathbf{M})$ as given in (8) and (9). But since the first approach of calculating overall 
Quick Identification of Stego Signatures in Images using Suspicion Value (special reference to pixel aberration i.e. $\boldsymbol{\delta}_{\mathbf{1}}(\mathbf{M})$ uses weights so even this suffers from the same distortions as the weighted mean pixel aberration $\boldsymbol{\delta}_{\mathbf{w}}(\mathbf{M})$ suffers. But the magnitude of $\boldsymbol{\delta}_{\mathbf{1}}(\mathbf{M})$ multiplied by 10 is a pretty good indicator of presence of information (stored using concentrating stego algorithms) in any image $\mathrm{M}$ and can be considered as the measure of Concentrating Suspicion Value $\boldsymbol{\Gamma}_{\mathbf{1}}(\mathbf{M})$ of image $\mathbf{M}$.

But even more accurate method of determining Concentrating Suspicion Value can be based on the combination of simple mean $\boldsymbol{\delta}_{\mathrm{m}}{ }^{\mathrm{MEAN}}(\mathbf{M})$ and overall Pixel Aberration $\boldsymbol{\delta}_{\mathbf{1}}(\mathbf{M})$. This combination can be done by finding the product of $\boldsymbol{\delta}_{\mathrm{m}}{ }^{\text {MEAN }}(\mathbf{M})$ and $\boldsymbol{\delta}_{\mathbf{1}}(\mathbf{M})$ and can be used as the second measure of concentrating suspicion value $\boldsymbol{\Gamma}_{2}(\mathbf{M})$ for any image $\mathbf{M}$. Thus the values $\boldsymbol{\Gamma}_{\mathbf{1}}(\mathbf{M})$ and $\boldsymbol{\Gamma}_{2}(\mathbf{M})$ of Images in Fig 2 is calculated in Table 4 and the algorithm for same is shown in (12). But the second measure of concentrating suspicion value $\boldsymbol{\Gamma}_{2}(\mathbf{M})$ suffers from much higher complexity and hence consumes far higher computation time and computation power.

Table 3

The values of $\delta_{1}(\mathbf{M})$ of the Cover Images $A$ and $B$ and the corresponding Stego Images

\begin{tabular}{|c|c|c|c|c|c|c|}
\hline Inage $\mathbf{M}$ & Description of M & $\delta_{1}^{\mathrm{R}}(\mathrm{M})$ & $\delta_{1}^{G}(\mathrm{M})$ & $\delta_{1}^{\mathrm{B}}(\mathrm{M})$ & $\delta_{1}^{\mathrm{MAX}}(\mathrm{M})$ & $\delta_{1}^{\text {MII } 1 \text { (M) }}$ \\
\hline$A$ & $\begin{array}{l}\text { Cover Image } \\
\text { (Fig 2, Left) }\end{array}$ & 1.0189 & 0.4276 & 0.5924 & 1.0159 & 0.6796 \\
\hline Al & Stego (Distributing 1) & 3.1412 & 0.3976 & 13.2676 & 13.2676 & 5.6022 \\
\hline A2 & Stego (Distributing 2) & 0.9668 & 0.3965 & 0.5554 & 0.96068 & 0.6396 \\
\hline$A 3$ & Stego (Concentrating) & 13595 & 1.0443 & 0.6211 & 1.3595 & 1.0083 \\
\hline B & $\begin{array}{l}\text { Cover Image } \\
\text { (Fig 2, Right) }\end{array}$ & 0.2260 & 0.2195 & 0.2324 & 0.2324 & 0.2260 \\
\hline $\mathrm{Bl}$ & Stego (Distributing 1) & 0.2354 & 0.2184 & 0.2421 & 0.2421 & 0.2320 \\
\hline B2 & Stego (Distributing 2) & 0.2297 & 0.2186 & 0.2367 & 0.2367 & 0.2283 \\
\hline B3 & Stego (Concentrating) & 1.2866 & 1.1435 & 0.2493 & 1.2866 & 0.5931 \\
\hline
\end{tabular}

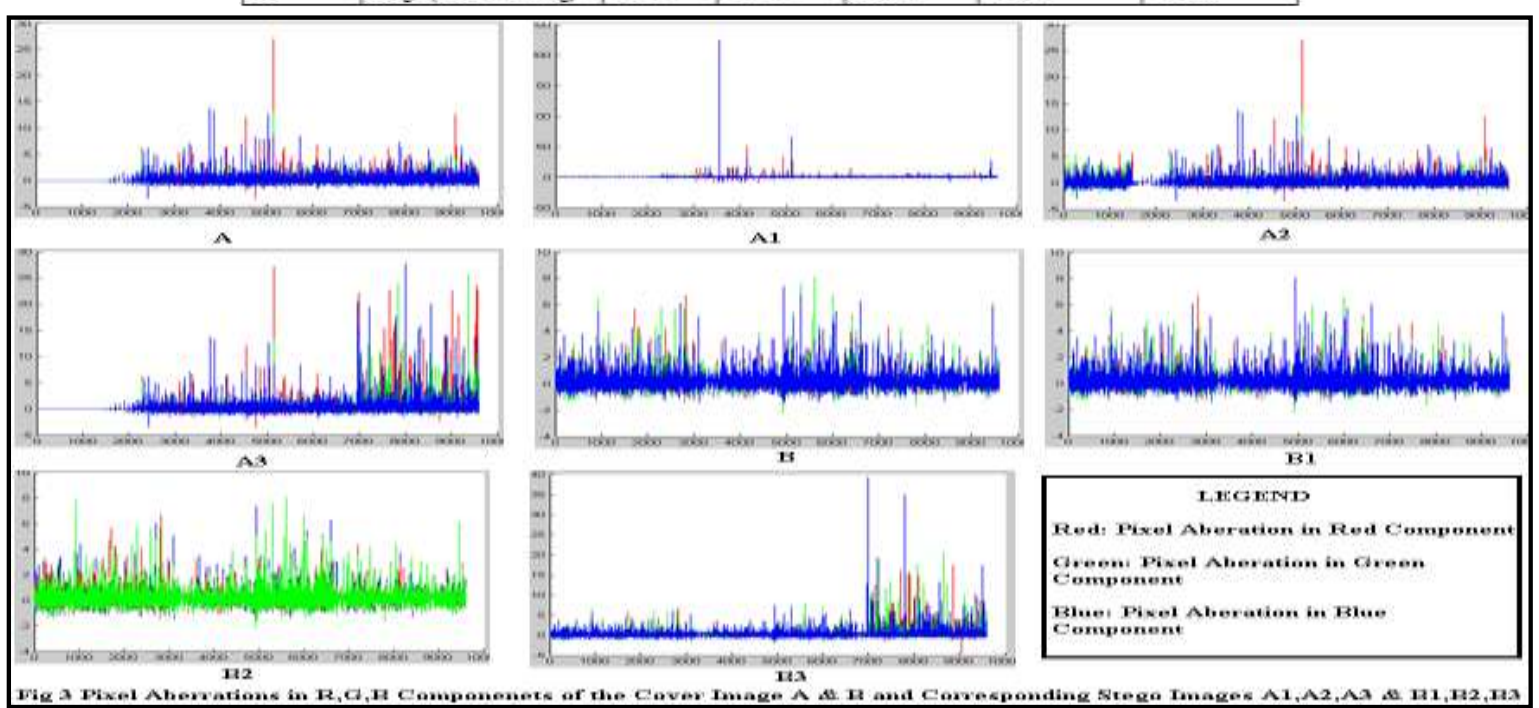

\subsubsection{Concentrating Suspicion Value Calculation by Second Approach}

The algorithm for calculating the Pixel Aberration for any given Image by combining the Simple and Weighted Means together by Second Approach is given in (13). On the basis of (13) the value of $\boldsymbol{\delta}_{\mathbf{2}}(\mathbf{M})$ is calculated for the same images (Fig 2) and shown in Table 5. Since the calculation of $\boldsymbol{\delta}_{2}(\mathbf{M})$ also uses Divide and Conquer Technique so like computation of $\boldsymbol{\delta}_{\mathbf{1}}(\mathbf{M})$ even its computation is much faster than the calculation of plain weighted mean pixel aberration for entire image i.e. $\boldsymbol{\delta}_{\mathbf{w}}(\mathbf{M})$ as given in (8) and (9). In Table 6 we have determined the value of Concentrating Suspicion Value of any Image M represented as $\boldsymbol{\Gamma}_{\mathbf{3}}(\mathbf{M})$ by using second approach based on $\boldsymbol{\delta}_{2}(\mathbf{M})$ of any image M. The algorithm for calculating $\boldsymbol{\Gamma}_{\mathbf{2}}(\mathbf{M})$ is given in (13). Table 4

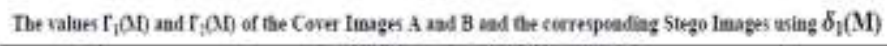

\begin{tabular}{|c|c|c|c|c|c|}
\hline Image M & Descriptice of $\mathbf{M}$ & $\delta_{1}^{\text {MEAS }}(\mathrm{N})$ & $\delta_{\mathrm{m}}^{\mathrm{sen} A \mathrm{X}}(\mathrm{M}$ & $\begin{array}{l}r_{1} A f_{1} \\
=\delta_{1}\end{array}$ & $\begin{array}{l}\mathrm{r} \\
\delta \\
\delta_{\mathrm{n}} \mathrm{MEN}^{1}(\mathrm{M}) \times 100\end{array}$ \\
\hline A & $\begin{array}{l}\text { Coter Image } \\
\text { (Fig } 2 \text { Left) }\end{array}$ & 0.6796 & 0.1139 & 6.796 & 07.7406 \\
\hline Al & Strgo (Distrilhuting 1 ) & 5.6522 & 01159 & 56.622 & 649295 \\
\hline A2 & Streo (Distrilluting 2) & 6.0396 & 0.1162 & 6.395 & 07.4322 \\
\hline A3 & Stego (Cobcentrating) & 1.0553 & 0.1562 & 16.053 & 15.7496 \\
\hline 8 & $\begin{array}{l}\text { Cover Image } \\
\text { fFys } 2 \text { Righon }\end{array}$ & 0.2260 & 0.1788 & 2.260 & 040409 \\
\hline B1 & Stego (Distniliveting 1) & 0.2280 & 0.1776 & 2330 & 04.1205 \\
\hline B: & Stkgo (Distrilhutias ?) & 0.2283 & 0.1783 & 2283 & 040706 \\
\hline B3 & Strego (Concentrating) & e.8931 & 0.2013 & 8931 & 181567 \\
\hline
\end{tabular}


The values of $\delta_{2}(\mathrm{M})$ of the Cover Innages $\mathrm{A}$ and $\mathrm{B}$ and the correspuding Stego Inages.

\begin{tabular}{|c|c|c|c|c|c|c|}
\hline Image M & Description of MI & $\delta_{2}^{R}(\mathrm{M})$ & $\delta_{2}{ }^{G}(\mathrm{M})$ & $\delta_{2}^{\mathrm{B}}(\mathrm{M})$ & $\delta_{z}^{3 L 2 X}(\mathrm{M})$ & $\delta_{2}^{3}{ }^{3}(\mathrm{M})$ \\
\hline A & $\begin{array}{l}\text { Coter Imags } \\
\text { (Fit 2, Leff) }\end{array}$ & 13362 & 0.9998 & 1.3516 & 13516 & 11992 \\
\hline Al & Stego (Distributing 1) & 2.2078 & 1.1126 & 2.4535 & 2.4555 & 1924 \\
\hline A2 & Stego (Distriburing 2) & 1.5978 & 1.1735 & 1.6165 & 10105 & 1.4613 \\
\hline A3 & Strgo (Concentrating) & 2.0362 & 1.5893 & 1.2786 & 2.6362 & 1.0243 \\
\hline B & $\begin{array}{l}\text { Covw Imagr } \\
\text { (Fig 2. Fighin) }\end{array}$ & 1.1592 & $1.172 \%$ & 1.1850 & 1.1864 & 1.1723 \\
\hline B1 & Strgo (Distributing 1) & 1.1560 & 1.1500 & 1.216 & 1.2100 & 1.1726 \\
\hline B2 & Stego (Distributing 2) & 1.1054 & 1.1812 & $1.20^{\circ} 2$ & 1.2072 & 11845 \\
\hline B3 & Stego (Concentrating) & 1.8136 & 1.9401 & 1.1279 & 19501 & 1.6305. \\
\hline
\end{tabular}

Table 6

The values $\Gamma_{2}(\mathrm{M})$ of Corer Images $A$ and $B$ and the corresponding Stego Inages using $\delta_{2}(M)$

\begin{tabular}{|l|l|l|l|}
\hline Image M & Description of M & $\delta_{2}^{\text {MIE.II }}(\mathrm{MI})$ & $\Gamma_{2}\left(\mathrm{MI}=\left(\delta_{2}^{\text {XIE. IV }}(\mathrm{M})-1.2\right) \times 100\right.$ \\
\hline A & $\begin{array}{l}\text { Cover Image } \\
\text { (Fig 2, Left) }\end{array}$ & 0.6796 & -0.0385 \\
\hline Al & Stego (Distributing 1) & 5.6022 & 36.236 \\
\hline A2 & Stego (Distributing 2) & 0.6396 & 13.065 \\
\hline A3 & Stego (Concentrating) & 1.0083 & 21.2168 \\
\hline B & $\begin{array}{l}\text { Cover Image } \\
\text { (Fig 2, Right) }\end{array}$ & 0.2260 & -1.3763 \\
\hline B1 & Stego (Distributing 1) & 0.2320 & -1.3684 \\
\hline B2 & Stego (Distributing 2) & 0.2283 & -0.7691 \\
\hline B3 & Stego (Concentrating) & 0.8931 & 21.5248 \\
\hline
\end{tabular}

Let the Intage to be analyzed be $\mathbf{M}$

The overall pixel Aberration by Furst Approach for Image $\mathbf{M}$ be $\boldsymbol{\delta}_{1}(\mathbf{M})$

Let image $\mathbf{M}$ be broken into $\mathbf{K}$ Components $\mathbf{M}^{\mathbf{1}}, \mathbf{M}^{2}, \mathbf{M}^{3}, \ldots . \mathbf{M}^{\mathbf{k}}$

size of Each Image Component is $\mathbf{n}\left(\mathbf{M}^{\mathbf{i}}\right)$

$$
\begin{aligned}
& \mathbf{M}=\bigcup_{\substack{i=1 \\
M^{i}=\mathbf{M}}}^{\mathbf{K}} \mathbf{M}^{\mathbf{1}}
\end{aligned}
$$

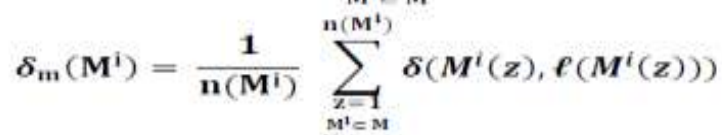

$$
\begin{aligned}
& s_{1}(M)-\frac{\sum_{M^{*}=M_{M}}^{N} s_{m}\left(M^{i}\right) \times w_{i}}{\sum_{M^{*}=M_{M}}^{K} w_{i}} \\
& W_{i}=\left|\frac{\delta_{m}\left(M^{i}\right)-M E A N_{i=1}^{K}\left(\delta_{m}\left(M^{i}\right)\right)}{\operatorname{STD}_{i=1}^{K}\left(\delta_{m}\left(M^{i}\right)\right)}\right|^{3} \\
& W_{i}=\left|\frac{\delta_{m}\left(M^{\prime}\right)-\frac{1}{K} \sum_{M=M}^{K} \delta_{m}\left(M^{i}\right)}{\sqrt{\frac{1}{K}\left(\sum_{M=M}^{K}\left(\delta_{m}\left(M^{\prime}\right)-\frac{1}{K} \sum_{M=M}^{K} \delta_{m}\left(M^{\prime}\right)\right)^{2}\right)}}\right|^{3}
\end{aligned}
$$

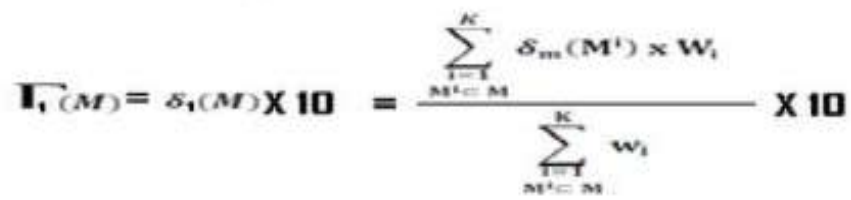

$$
\begin{aligned}
& T_{2}(M)={ }_{m}(M) \quad X \quad \delta_{1}(M) \times 100
\end{aligned}
$$

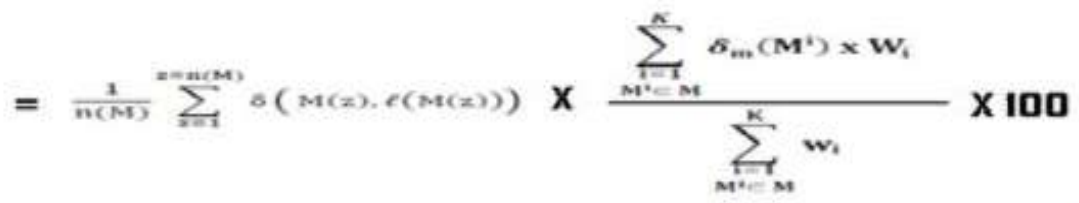




$$
\begin{aligned}
& \text { Let the Image to be analyzed be } \mathbf{M} \\
& \text { The overall pixel Aberration of Image } \mathbf{M} \text { be } \boldsymbol{\delta}(\mathbf{M}) \\
& \text { Let Image } \mathbf{M} \text { be broken into } \mathbf{K} \text { Components } \mathbf{M}^{1}, \mathbf{M}^{2}, \mathbf{M}^{3}, \ldots ., \mathbf{M}^{\mathbf{K}} \\
& \text { Size of Each Image Component is } \mathbf{n}\left(\mathbf{M}^{\mathbf{i}}\right) \\
& \mathbf{M}=\bigcup_{\substack{\mathrm{i}=1 \\
\mathbf{M}^{\mathrm{i}} \subset \mathbf{M}}}^{\mathrm{K}} \mathbf{M}^{\mathrm{i}} \\
& \delta_{\mathbf{w}}\left(\mathbf{M}^{\mathbf{i}}\right)=\frac{\sum_{\substack{z=1 \\
M^{i}(z) \in M^{i} \\
M^{i} \subset M}}^{n\left(M^{i}\right)}\left|\delta\left(M^{i}(z), \ell\left(M^{i}(z)\right)\right)\right| \times w^{i}(z)}{\sum_{\substack{z=1 \\
M(z) \in M}}^{N} w^{i}(z)} \\
& \mathrm{W}_{\mathrm{i}}(\mathrm{z})=\left|\frac{\delta\left(M^{i}(\mathrm{z}), \mathcal{\ell}\left(M^{i}(\mathrm{z})\right)\right)-\operatorname{MEAN}_{\mathrm{i}=1}^{\mathrm{K}}\left(\delta\left(M^{i}(\mathrm{z}), \ell\left(M^{i}(\mathrm{z})\right)\right)\right)}{\operatorname{STD}_{\mathrm{i}=1}^{\mathrm{K}}\left(\delta\left(M^{i}(\mathrm{z}), \ell\left(M^{i}(\mathrm{z})\right)\right)\right)}\right|^{3} \\
& \text { OR }
\end{aligned}
$$

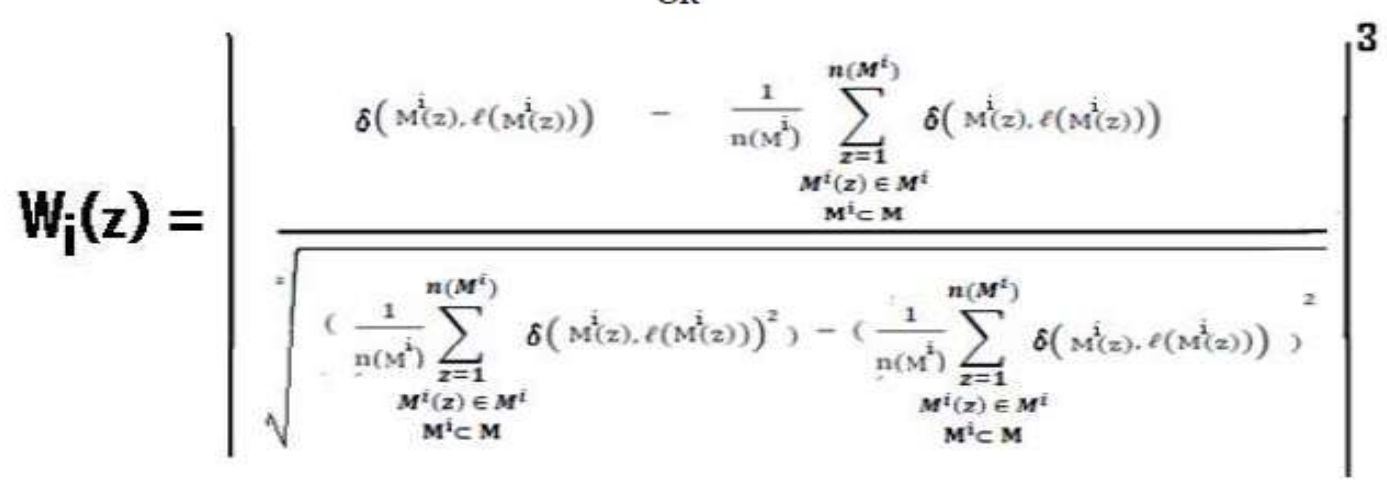

$$
\begin{aligned}
& \delta_{2}(\mathbf{M})=\frac{1}{\mathbf{K}} \sum_{\substack{\mathrm{i}=1 \\
\mathbf{M}^{1} \subset \mathrm{M}}}^{\mathrm{K}} \delta_{\mathrm{W}}\left(\mathbf{M}^{\mathrm{i}}\right) \\
& \Gamma_{3}(M)=\left(\delta_{2}(M)-1.2\right) \times 100
\end{aligned}
$$

\subsubsection{Concentrating Suspicion Value $\Gamma(M)\left(\right.$ Combining $\Gamma_{1}(M), \Gamma_{2}(M)$ and $\Gamma_{3}(M)$ in to single value)}

In Section 2.3.2.1 and Section 2.3.2.2 the Concentrating Suspicion Value of any Image has been calculated by three different methods using (12) and (13) respectively and represented as $\boldsymbol{\Gamma}_{\mathbf{1}}(\mathbf{M}), \boldsymbol{\Gamma}_{\mathbf{2}}(\mathbf{M})$ and $\boldsymbol{\Gamma}_{\mathbf{3}}(\mathbf{M})$ respectively. From (12) and (13) it can be clearly concluded that the Complexity of determining $\boldsymbol{\Gamma}_{\mathbf{2}}(\mathbf{M})$ is far higher than $\boldsymbol{\Gamma}_{\mathbf{1}}(\mathbf{M})$ or $\boldsymbol{\Gamma}_{\mathbf{3}}(\mathbf{M})$. Hence calculation of suspicion value using $\boldsymbol{\Gamma}_{2}(\mathbf{M})$ is ruled out in all circumstances. The performance of the two different types of concentrating suspicion values $\boldsymbol{\Gamma}_{\mathbf{1}}(\mathbf{M})$ and $\boldsymbol{\Gamma}_{\mathbf{3}}(\mathbf{M})$ is examined by analyzing four different test images acting as cover images and corresponding Stego Images obtained by two different stego softwares. These two softwares are used for embedding same information (1900 Characters) in all the four images as shown in Fig 4. These images (Fig 4) are of dimensions 600x800, $275 \mathrm{x}$ $181,600 \times 800$ and $340 \times 506$ pixels and represented as A, B, C and D respectively. One of the two softwares produces Stego Image by using Distributing Stego Algorithm while the other produces Stego image by using Concentrating Stego Algorithm. The Stego Images produced by Distributing Stego Algorithms are represented as A_d ,B_d ,C_d and D_d while the Stego Images produced by Concentrating Stego Algorithms are represented as A_c, B_c, C_c and D_c. 


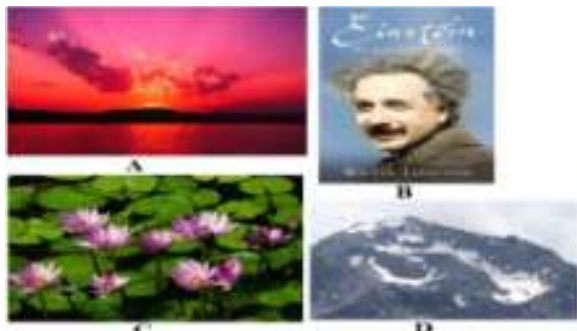

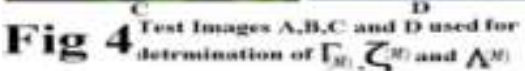

Table?

The Valaes of $\left.\mathrm{r}_{1} \mathrm{AD}, \mathrm{T}, \mathrm{M}\right)$ and $\mathrm{r}(\mathrm{M})$ of four differeut Cover Images $(\mathrm{A}, \mathrm{B}, \mathrm{C}$ and $\mathrm{D})$

and the correspouding Stege Images

\begin{tabular}{|c|c|c|c|c|}
\hline $\begin{array}{l}\text { Image } \\
\mathrm{M}\end{array}$ & Image Properties & $\mathrm{r}_{\mathrm{B}}(\mathrm{M})$ & r, $\left(D_{1}\right.$ & $\begin{array}{l}\mathrm{r}(\mathrm{A})= \\
\mathrm{MAX}\left(\mathrm{r}_{1}, \mathrm{M}, \mathrm{r}_{l}(\mathrm{AM})\right.\end{array}$ \\
\hline A & $600 x 500$ Pixel Cover & 2.192 & -17.3155 & 2.192 \\
\hline A d & Distributing & 2.1966 & -16.0829 & 2.1966 \\
\hline A $c$ & Concentrating & 20.3353 & -6.0160 & 20.3353 \\
\hline $\mathrm{B}$ & 275xl81 Pixel Coser & 4.5153 & -12.1805 & 4.5153 \\
\hline B d & Distributing & 4.5346 & -10.389 & 4.5346 \\
\hline$B_{C} C$ & Concentrating & 261.0982 & 184.8828 & 261.0982 \\
\hline C & 6005500 Pixel Co5er & 1.909 & -25.7871 & 1.909 \\
\hline C d & Distributing & 1.9708 & -24.4523 & 1.9708 \\
\hline$C_{C} \mathrm{C}$ & Concentrating & 53.8520 & -12.9077 & 53.8520 \\
\hline D & $340 x 506$ Pixel Cover & 2.8924 & -4.6067 & 2.8924 \\
\hline D_d & Distributing & 2.9313 & -4.2348 & 2.9313 \\
\hline D C & Concentrating & 20.0985 & -1.9733 & 20.0985 \\
\hline
\end{tabular}

From Table 7 it can be easily concluded that $\boldsymbol{\Gamma}_{3}(\mathbf{M})$ is the true measure of Concentrating Suspicion Value $\boldsymbol{\Gamma}(\mathbf{M})$ of any Image M. The same can be mathematically written as:

$$
\Gamma(M)=\Gamma_{3}(M)
$$

\subsection{Determination of Overall Suspicion Value $\zeta$}

The values of Concentrating Suspicion Value $\boldsymbol{\Gamma}(\mathbf{M})$ as obtained from Table 7 is combined with the Distributing Suspicion Value $\boldsymbol{\Lambda}(\mathbf{M})$ (determined from (17) in [8]) to Produce Overall Suspicion Values $\boldsymbol{\zeta}(\mathbf{M})$ for the four different test images in Fig 4 (A,B,C and D) and the corresponding Stego Images(A_d ,B_d ,C_d and D_d ;A_c, B_c, C_c and D_c). The same is shown in Table 8. Thus we see that Overall Suspicion Value $\zeta(\mathbf{M})$ is very much higher for all the images having hidden information while it is much lower for the innocent cover images. The Overall Suspicon Value $\zeta(\mathbf{M})$ for any image $\mathbf{M}$ is the maximum of the Concentrating and Distributing Suspcion Values and mathematically given in (15).

$$
\zeta(\mathbf{M})=\operatorname{MAX}(\Gamma(\mathbf{M}), \Lambda(\mathbf{M}))
$$

Table 8

The Values of $\mathrm{r}(\mathrm{M}), \Lambda(M)$ and $\zeta(\mathrm{M})$ of four different Cover Images (A, B, C and D)

\begin{tabular}{|c|c|c|c|c|}
\hline $\begin{array}{l}\text { Image } \\
\text { M }\end{array}$ & Image Properties & $\Gamma(\mathrm{AI})$ & $\Lambda(\mathrm{M})$ & $\zeta(\mathrm{M})=\mathrm{MAX}(\mathrm{\Gamma}(\mathrm{M}), \Lambda(\mathrm{M}))$ \\
\hline A & $600 \times 800$ Pixel Cover & 2.192 & 3.0581 & 3,0581 \\
\hline A d & Distributing & 2.1966 & 43.9658 & 43.9658 \\
\hline A c & Concentrating & 20.3353 & 3.0558 & 20.3353 \\
\hline B & 275x181 Pixel Cover & 4.5153 & 2.1899 & 4.5153 \\
\hline B d & Distributing & 4.5346 & 17.9565 & 17.9565 \\
\hline B_c & Concentrating & 261.0982 & 2.1899 & 261.0982 \\
\hline $\mathrm{C}$ & $600 x 800$ Pixel Cover & 1.909 & 3.2240 & 3.2240 \\
\hline C_d & Distributing & 1.9708 & 34.3697 & 34.3697 \\
\hline $\mathrm{C}_{\mathrm{C}}$ & Concentrating & 53.8520 & 3.2858 & 53.8520 \\
\hline $\mathbf{D}$ & $340 x 506$ Pixel Cover & 2.8924 & 1.2039 & 2.8924 \\
\hline D_d & Distributing & 2.9313 & $\begin{array}{l}0.8429 \\
829 \\
\text { Ambiguous } \\
\text { Section }\end{array}$ & $\begin{array}{l}\text { Ambiguous Section when } \\
\text { analyzed in detail clearly } \\
\text { shows the presence of } \\
\text { information embedded in } \\
\text { those sections. }\end{array}$ \\
\hline D c & Concentrating & 20.0985 & 1.2039 & 20.0985 \\
\hline
\end{tabular}
and the corresponding Stego Images 


\section{CONCLUSION}

The Distributing Suspicion Value (obtained from (17) in [8]) and Concentrating Suspicon Value (obtained from (12), (13) and (14)) are combined together using (15) to produce Overall Suspicion Value $\zeta(\mathrm{M})$ associated with any given image M. From Table 8 it can be clearly seen that this numerical quantifier $\zeta(M)$ is higher for all those images which have some information embedded in them while it is much lower for all innocent cover images. Hence this holistic suspicion value $\zeta(\mathrm{M})$ (which applies on both the Concentrating as well as Distributing Stego Algorithms) is a quick identifier of presence of information in any given image and can be effectively used as Stego Identifier Algorithm. This fast stego-identification technique will find its application in quick filtering of the suspicious images flowing through the web servers, routers, layer three switches and all other electronic media concerned with transmission of images and will be very useful tool against terrorists and all other mala-fide cyber networks.

\section{ACKNOWLEDGEMENT}

I wish to dedicate this work to my parents Mr Chandan Kumar Choudhary and Mrs Nilima Choudhary for providing the necessary support and encouragement in all walks of life including this work.

\section{REFERENCES}

[1]. Infosecurity Magazine article dated 02 May 2012 reports that Al-Qaeda uses Steganography to hide documents. http://www.infosecurity-magazine.com/view/25524/alqaeda-uses-steganography-documents-hidden-in-porn-videos-found-onmemory-stick

[2] Daily Mail Online, UK article dated 01 May 2012 reported that a Treasure trove of Intelligence was embedded in porn video.http://www.dailymail.co.uk/news/article-2137848/Porn-video-reveals-Al-Qaeda-planns-hijack-cruise-ships-executepassengers.html\#ixzzluIgxpire

[3] The New York Times article dated Oct 30, 2001 with title "Veiled Messages of Terror May Lurk in Cyberspace" claims 9/11 attacks planned using Steganography.

[4] Wired article dated $02^{\text {nd }}$ July, 2001 nicknamed Bin Laden as "the Steganography Master"http://www.wired.com/politics/law/news/2001/02/41658?currentPage=all

[5] Kaustubh Choudhary, Image Steganography and Global Terrorism, IOSR Volume 1, Issue 2, July 2012. http://iosrjournals.org/journals/iosr-jce/papers/vol1-issue2/14/N0123448.pdf

[6] Kaustubh Choudhary, Mathematical Modeling of Image Steganographic System IOSR Volume 2, Issue 5, August 2012 http://iosrjournals.org/journals/iosr-jce/papers/vol2-issue5/A0250115.pdf

[7] Kaustubh Choudhary, Novel Approach to Image Steganalysis (A Step against Cyber Terrorism) IOSR Volume 2, Issue 5, August 2012 http://iosrjournals.org/journals/iosr-jce/papers/vol2-issue5/B0251628.pdf

[8] Kaustubh Choudhary, Identification of Steganographic Signatures of Distributing Stego Algorithms using Suspicion Value , IOSR Volume 3, Issue 4, August 2012

[9] Kaustubh Choudhary, Identification of Stego Signatures in Images using Suspicion Value (special reference to Concentrating Stego Algorithms), IOSR Volume 3, Issue 4, August 2012

\section{About THE AUTHOR}

Kaustubh Choudhary is Scientist in Defence Research and Development Organisation
(DRDO), Ministry of Defence, Government of India. He is currently attached with Indian
Navy at Indian Naval Ship, Shivaji as a faculty member of Naval College of Engineering.
He is young and dynamic scientist and has more than 5 Years of Experience in Teaching
and Research.

